\title{
Challenges in Conducting and Completing the Orthopaedic Postgraduate Thesis During COVID-19 Pandemic
}

\author{
Vijay Kumar Jain ${ }^{1} \cdot$ Deepak Gautam ${ }^{2}\left(\mathbb{D} \cdot\right.$ Karthikeyan P. lyengar $^{3} \cdot$ Raju Vaishya $^{4} \cdot$ Rajesh Malhotra $^{2}$
}

Received: 15 June 2021 / Accepted: 24 August 2021 / Published online: 14 September 2021

(c) Indian Orthopaedics Association 2021

\section{Background}

Thesis or dissertation writing is an integral part of postgraduate (PG) training programs for all medical specialties, including orthopaedics. The timing or the study duration is usually limited, and the candidate has to complete and submit his or her thesis work within the specified, limited period. The required complexity or quality of research of a thesis or dissertation can vary by country, university, or the training program itself. The residency program is for usually of three years' duration (comprising six semesters of six months each) in India. During the process of thesis preparation and submission, the PG students are exposed to an essential skills of Research (and possible future Publication). It offers them a unique and valuable opportunity to investigate a research topic related to their specialty in great depth. The thesis hones the candidate's writing skills, enhances awareness of the field of interest, and fuels ambition to learn.

Deepak Gautam

cmcdeepak@yahoo.com

Vijay Kumar Jain

drvijayortho@gmail.com

Karthikeyan P. Iyengar

kartikp31@hotmail.com

Raju Vaishya

raju.vaishya@gmail.com

Rajesh Malhotra

rmalhotra62@hotmail.com

1 Department of Orthopaedics, Atal Bihari Vajpayee Institute of Medical Sciences, Dr. Ram Manohar Lohia Hospital, New Delhi 110001, India

2 Department of Orthopaedics, All India Institute of Medical Sciences (AIIMS), New Delhi 110029, India

3 Southport and Ormskirk NHS Trust, Southport PR8 6PN, UK

4 Department of Orthopaedics, Indraprastha Apollo Hospital, Sarita Vihar, Mathura Road, New Delhi 110076, India
The ultimate value of a thesis is achieved when the research work is published in a scientific journal and complements to the global pool of knowledge.

\section{Problems Faced by the Postgraduates Related to Thesis During the COVID-19 Pandemic}

PG students are allocated a research topic by their thesis guides soon after joining the master's course. The COVID19 pandemic has seen a very turbulent course in the current times. The hospitals are being converted into COVID facilities, where the non-COVID patients are not being accommodated due to resource constraints, and the routine clinical work is interrupted. Therefore, the assigned clinical research work for the PG students is primarily not possible or severely compromised. COVID-19 pandemic has severely disrupted the postgraduate orthopedic education and training program [1]. Upadhyaya et al. have shown in a survey-based study that nearly $71.6 \%$ of PGs had problems completing their dissertations [1]. Most of the trainees have felt the reduction in clinical, surgical, and educational activities due to COVID19 would have a detrimental effect on their training [2].

The main problem in completing the thesis work is the allocation of patients, as there is a significant reduction in the patient load in the hospitals. Due to COVID-19, most of the centers have either stopped outpatient services or have reduced the timing of OPDs. Moreover, the patients enrolled in the research are not available for follow-up due to nonavailability of outpatient services as well as patients' fear of the visiting hospital during pandemic.

Another issue is the redeployment of orthopedic residents to the frontline of COVID duties, primarily in non-surgical roles in COVID areas such as triage, intensive treatment units, accident and emergency, and general medicine, etc. [3]. This directly affects the PG student, as they are taken away from the main orthopaedic stream. They cannot 
allocate, perform surgery or participate and follow-up with patients.

Candidates with a thesis on clinical aspects of orthopaedics such as arthroplasty, arthroscopic and spine surgery, deformity correction, soft tissue deformities, paediatric orthopaedic conditions are the worst affected. The candidates with a thesis on acute trauma are also suffering, albeit to a lesser degree, as the operating time in most of the hospitals is reduced due to the migration of population, Janta curfew, and, mini lockdown in many states. Many surgeons still operate the cases requiring urgent management such as compartment syndrome, septic arthritis, acute osteomyelitis, obligatory fractures (e.g., neck of femur), cauda equina, fractures with neurological deficits, and orthopaedic oncology despite COVID-19 as a priority.

Furthermore, unexpected sickness due to COVID-19 of the PG or their guide is another crucial factor; that may affect the thesis work. Follow-up of patients is difficult due to the deaths of patients and migration of the population.

Due to sub-optimal thesis work during COVID-19, future publication prospects of their research are further jeopardized. The inability to complete the thesis work satisfactorily amid COVID-19 could cause distress (stress and anxiety) and negatively affect the mental health of most candidates.

\section{Suggested Solutions to the Problems}

During COVID-19, the universities must give some special exemptions to the candidates related to their thesis. We suggest the following solutions for overcoming these problems:

- Instead of a clinical research topic, non-clinical research, such as systematic reviews, meta-analysis etc. could be allocated.

- The required sample size and/or follow-up duration of the cases be reduced especially those who had already started working in their allocated thesis topic/or are midway. However, the research work initiated can be continued to fulfill the cohort size and desired follow-up by allotting the same topic with some difference in the outcomes to residents from subsequent batches to complete the research.

- The research topics can be changed from surgical to nonoperative.

- The duration of enrolment, including allocation of patients can be prolonged in special conditions. This will reduce the follow-up period. The candidate, however, can continue to follow-up these patients after qualifying MS if they join as a Registrar/Senior Resident.

- Topics related to the effect of COVID-19 on musculoskeletal (MSK) disease, and orthopaedic care delivery can be given
- Allocation of topics on acute conditions, which are being managed during the COVID times, like orthopaedic oncology and MSK infection.

- Thesis on diagnostic studies related to orthopedic conditions could also be another option.

- Comparison of the proposed test or investigation with the GOLD standard test/investigation will show the diagnostic accuracy of the proposed test against the standard GOLD test/investigation

Example: New ELISA test with traditional culture and microbiological confirmation of bone TB. Further the diagnostic study evaluates the sensitivity, specificity, positive predictive value (PPV), negative predictive value (NPV0 and diagnostic accuracy of a NEW/innovative or proposed test against the GOLD standard can be given as thesis topics.

\section{Conclusion}

COVID-19 pandemic has negatively affected healthcare delivery and directly impacted the PG training of the medical student especially that of orthopaedics. The PGs face tremendous and multiple challenges in completing their thesis work in the stipulated time of their curriculum. We have suggested the solutions to overcome the problems of PGs in completing their thesis for the master's degree.

Acknowledgements None.

Funding The authors have not declared a specific grant for this research from any funding agency in the public, commercial or not-for-profit sectors.

\section{Compliance with ethical standards}

Conflict of interest Nothing to disclose. "The authors declare no conflict of interest".

Ethical standard statement This article does not contain any studies with human or animal subjects performed by the any of the authors.

Informed consent For this type of study, informed consent is not required.

Patient consent for publication Not required.

\section{References}

1. Upadhyaya, G. K., Jain, V. K., Iyengar, K. P., Patralekh, M. K., \& Vaish, A. (2020). Impact of COVID-19 on post-graduate orthopaedic training in Delhi-NCR. Journal of Clinical Orthopaedics and Trauma, 11(Suppl 5), S687-S695.

2. Megaloikonomos, P. D., Thaler, M., Igoumenou, V. G., Bonanzinga, T., Ostojic, M., Couto, A. F., Diallo, J., \& Khosravi, I. 
(2020). Impact of the COVID-19 pandemic on orthopaedic and trauma surgery training in Europe. International Orthopaedics, 44(9), 1611-1619.

3. Gonzi, G., Gwyn, R., Rooney, K., Boktor, J., Roy, K., Sciberras, N. C., Pullen, H., \& Mohanty, K. (2020). The role of orthopaedic trainees during the COVID-19 pandemic and impact on postgraduate orthopaedic education: A four-nation survey of over 100 orthopaedic trainees. Bone \& Joint Open, 1(11), 676-682.
Publisher's Note Springer Nature remains neutral with regard to jurisdictional claims in published maps and institutional affiliations. 